\title{
Massive star populations in Lyman break galaxies
}

\author{
Max Pettini, Samantha A. Rix \\ Institute of Astronomy, Madingley Road, Cambridge CB3 OHA, UK \\ Charles C. Steidel, Alice E. Shapley \\ Palomar Observatory, Caltech 105-24, Pasadena, CA 91125, USA
}

Kurt L. Adelberger

Center for Astrophysics, 60 Garden Street, Cambridge, MA 02138, USA

\begin{abstract}
Massive stars provide most of our current knowledge of the first star forming galaxies in the universe via their ultraviolet and optical spectra redshifted into the visible and near-infrared bands. Here I briefly review the growing body of data on these early stellar populations from which star formation rates, the initial mass function, chemical abundances, ages, and dust reddenings are being deduced. New evidence also shows the dramatic impact which massive stars have on their galactic and intergalactic environments.
\end{abstract}

\section{Introduction}

This is the first time I have taken part in one of the meetings in this series which began many years ago. While until recently the study of massive stars was limited to our Galaxy and the nearby universe, the last few years have witnessed a major leap forward in our capabilities to extend this field of research to very high redshifts. The integrated light of young stellar populations from distant galaxies, and of their associated $\mathrm{H}$ II regions, is now routinely detected up to $z \simeq 4.5$, and even beyond. The current record holder is a galaxy at $z=6.56$ (Hu et al. 2002); the Ly $\alpha$ photons which we collect with telescopes on Earth were emitted by this object when the universe was less than $1 \mathrm{Gyr}$ old ${ }^{1}$. The study of massive stars has definitely acquired a new relevance for observational cosmologists.

The largest body of data on 'normal' galaxies at high redshifts pertains to the Lyman break galaxy (LBG) population at $z \simeq 3$. A typical deep exposure at the prime focus of a $4 \mathrm{~m}$ telescope reveals 100 - 200 such objects brighter than $R=25.0-25.5 \mathrm{mag}$ in a $10^{\prime} \times 10^{\prime}$ random patch of sky (at high Galactic latitudes); they are straightforward to recognise from their colours, which are markedly

${ }^{1}$ In a $H_{0}=65 \mathrm{~km} \mathrm{~s}^{-1} \mathrm{Mpc}^{-1}, \Omega_{\mathrm{M}}=0.3, \Omega_{\Lambda}=0.7$ cosmology. 


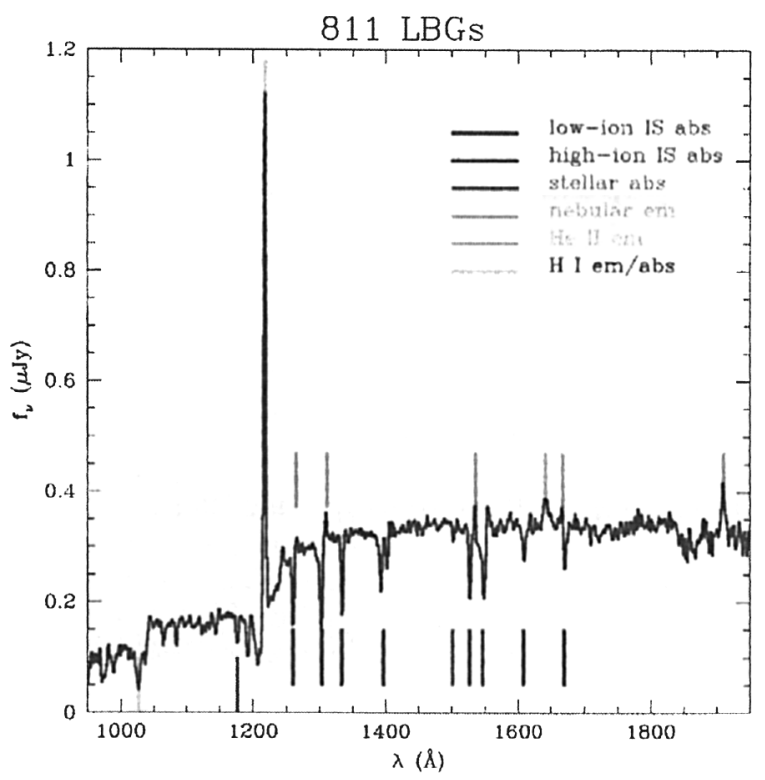

Figure 1. 'Typical' rest-frame UV spectrum of a Lyman break galaxy, obtained by co-adding 811 individual spectra to improve the signal-to-noise ratio.

different from those of lower redshift galaxies (Steidel, Pettini \& Hamilton 1995). Thanks to the multi-object capabilities and efficiency of modern spectrographs on $8-10 \mathrm{~m}$ telescopes, such as LRIS on Keck I, it has been possible to build up large samples of spectroscopically confirmed LBGs in only a few years. Our own survey has yielded nearly one thousand galaxies in the interval $2.5 \lesssim z \lesssim 3.5$, from which the overall properties of the population - most importantly their luminosity function and large-scale distribution and clustering - have been determined (see Steidel 2000 for a review).

When we record the spectrum of a $z \simeq 3$ galaxy at optical wavelengths we observe the rest-frame ultraviolet (UV) light from the O- and B-type stars, redshifted into the visible window. The 'typical' UV spectrum of a Lyman break galaxy is best illustrated by adding together all the individual spectra in the sample (having determined the systemic redshift of each as carefully as possible and shifting to a common wavelength scale), as done by Shapley et al. (2003).

As can be seen from Figure 1, the UV spectra of LBGs are a composite of many features, including blends of photospheric lines, P-Cygni emission and absorption by the most luminous massive stars, narrower emission lines from $\mathrm{H}$ II regions, and resonance absorption lines from interstellar gas in a wide range of ionisation stages. By analysing these different signatures we can address a number of issues of relevance to the subject of this meeting. Some of the questions which we would like to answer are:

(i) what is the nature of the stellar populations we see in Lyman break galaxies;

(ii) to what level have they been enriched in heavy elements;

(iii) what are their ages and masses; and 


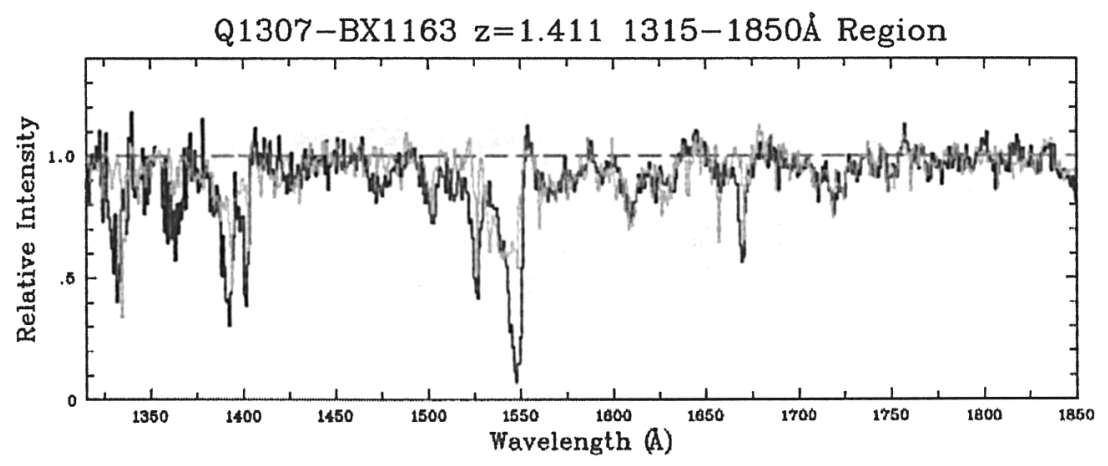

Figure 2. Q $1307-\mathrm{BX} 1163$ is a recently discovered bright $(R=21.78 \mathrm{mag})$ star-forming galaxy $\left(\mathrm{SFR} \simeq 50 \mathrm{M}_{\odot} \mathrm{yr}^{-1}\right)$ at $z=1.411$. Here we compare its rest-frame far-UV spectrum (black histogram, recorded at a resolution of $2.5 \AA$ FWHM, with the newly commissioned blue arm of LRIS on Keck I) with a STARBURST99 model for $100 \mathrm{Myr}$-old continuous star formation with a Salpeter IMF and solar metallicity (grey histogram).

(iv) what are the effects of star formation at high $z$ on the galaxies and the surrounding intergalactic medium (IGM)?

We illustrate each of these points with one example, chosen to give a flavour of the active work in progress in all of these areas.

\section{Stellar populations and the Initial Mass Function (IMF)}

The most direct information on these topics is obtained by comparing the spectra of a few LBGs which have been recorded at high spectral resolutions and signalto-noise ratios with model spectra generated by spectral synthesis codes such as STARBURST99 (Leitherer et al. 1999). From the example reproduced in Figure 2 it can be appreciated how closely the integrated light from massive stars in this distant galaxy resembles that of Population I stars in the Milky Way. The numerous blends of photospheric lines, and the P-Cygni components of CIV $\lambda 1549$ and Si IV $\lambda 1397$, are reproduced very well by a continuous episode of star formation with a Salpeter slope at the upper end of the IMF and solar metallicity. The only significant difference between the observed and calculated spectra is in the strengths of the interstellar absorption lines (which STARBURST99 does not attempt to reproduce). The fact that they are much stronger in Q 1307-BX1163 than in the Milky Way is probably an indication that the interstellar medium of this starburst galaxy at $z=1.411$ has been stirred to much higher velocity dispersions than the more quiescent gas near the Sun, probably as a result of the high level of star formation activity. This is a common situation in LBGs.

The wind lines of C IV and Si IV (as well as Nv $\lambda 1240$ which is not covered in our spectrum of Q 1307-BX1163) are particularly sensitive to the upper end of the IMF. As can be seen from Figure 3, even small changes to the IMF produce detectable differences in the emission and absorption components of the profiles, so that we can exclude, in this starburst, both a flatter IMF (relative to Salpeter) 

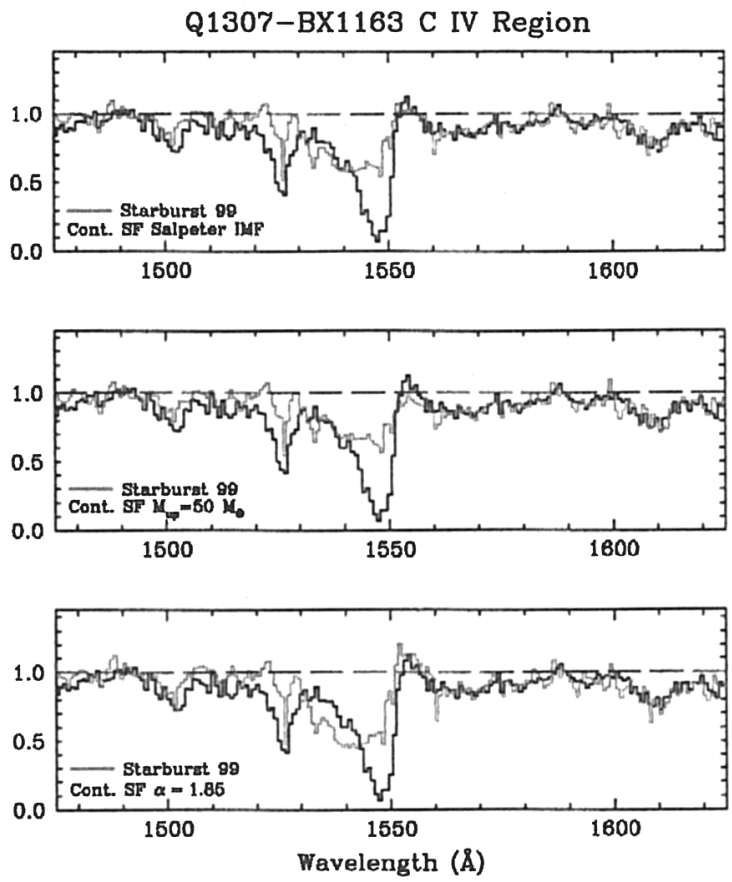

Figure 3. Comparisons between STARBURST99 spectral synthesis models (grey histograms) for different IMFs (all with solar metallicity), and the Keck spectrum of Q 1307-BX1163 (black histograms) in the region near the CIV doublet. The $y$-axis is residual intensity. A standard Salpeter IMF with slope $\alpha=-2.35$ and extending to $M_{\mathrm{up}}=100 \mathrm{M}_{\odot}$ gives the best fit to the P-Cygni components of the CIV line. Models in which the IMF either lacks (middle panel) or has an excess (bottom panel) of massive stars produce less satisfactory fits. The core of the CIV line is due to interstellar absorption, which is not accounted for in STARBURST99.

and one lacking in stars more massive than $50 \mathrm{M}_{\odot}$. Similar conclusions were reached by Pettini et al. (2000) for another well-studied LBG, MS 1512-cB58 at $z=2.7276$.

\section{Metals in Lyman break galaxies}

The P-Cygni wind lines are also sensitive to the metallicity of the O-and B-type stars, since it is through absorption and scattering of photons in metal transitions that momentum is transferred to the gas and a wind is generated. The STARBURST99 model spectra shown in Figures 2 and 3 are for solar metallicity and the good match to the observed spectrum of Q 1307-BX1163 suggests that this galaxy had already reached approximately solar abundances $10 \mathrm{Gyr}$ ago.

For MS 1512-cB58, which we observe at the earlier look-back time of $12 \mathrm{Gyr}$, we deduce a metallicity of approximately $1 / 3$ solar from similar considerations (Figure 4). This conclusion is further supported by measurements of element 
MS $1512-$-B58 C IV Region Keck II+ESI $1.4 \AA$ FWHM

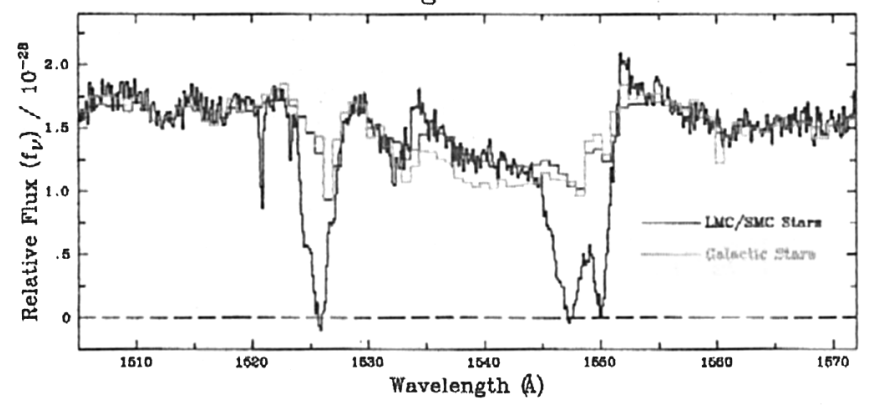

Figure 4. A portion of the high resolution spectrum of MS1512-cB58 obtained by Pettini et al. (2002), encompassing the C IV $\lambda \lambda 1548,1550$ doublet lines, is compared with STARBURST99 model spectra for solar and $\sim 1 / 3$ solar (LMC/SMC) metallicities (Leitherer et al. 2001). Both cases are for a standard Salpeter IMF. Sub-solar metallicities provide a better fit to the P-Cygni components of the $\mathrm{C}$ IV doublet; the small amount of residual emission above the model predictions is probably due to nebular C IV emission from the $\mathrm{H}$ II regions in the galaxy, superposed on the broader stellar P-Cygni profile.

abundances from interstellar absorption lines (Pettini et al. 2002). As can be seen from Figure 5, elements released by TypeII supernovae $(\mathrm{O}, \mathrm{Mg}, \mathrm{Si}, \mathrm{P}$, and S) have abundances of $\sim 2 / 5$ solar. Thus, even at this relatively early epoch, MS 1512-cB58 had already processed more than one third of its gas into stars. Furthermore, this galaxy appears to be chemically young, in that it is relatively deficient in elements produced by stars of intermediate and low masses, which have longer evolutionary timescales than those of Type II SN progenitors. $\mathrm{N}$ and the Fe-peak elements we observe $(\mathrm{Mn}, \mathrm{Fe}$, and $\mathrm{Ni}$ ) are all less abundant than expected by factors of between 0.4 and 0.75 dex. On the basis of current ideas of the nucleosynthesis of $\mathrm{N}$, it would appear that much of the ISM enrichment in MS 1512-cB58 has taken place within the last $250 \mathrm{Myr}$, the lifetime of the intermediate mass stars believed to be the main source of N. Taken together, these findings are highly suggestive of a galaxy caught in the act of converting its interstellar medium into stars on a few dynamical timescales - quite possibly we are witnessing the formation of a galactic bulge or an elliptical galaxy.

Such precise determinations of metallicity, from either the wind lines of massive stars or interstellar absorption lines, are possible only for a few LBGs which are either gravitationally lensed or at the bright end of the luminosity function. Measurements from $\mathrm{H}$ II regions are also difficult, because the familiar nebular emission lines are redshifted into the near-IR, where the airglow emission from the night sky can swamp them (Pettini et al. 2001). For this reason, we are currently exploring the possibility of using the equivalent widths of blends of photospheric lines to derive abundance sensitive indices in the UV. Initial results by Leitherer et al. (2001) are encouraging in that the $1425 \AA$ index defined by these authors has values for MS 1512-cB58 and Q 1307-BX1163 which are consistent with the metallicities deduced from more conventional diagnostics. 


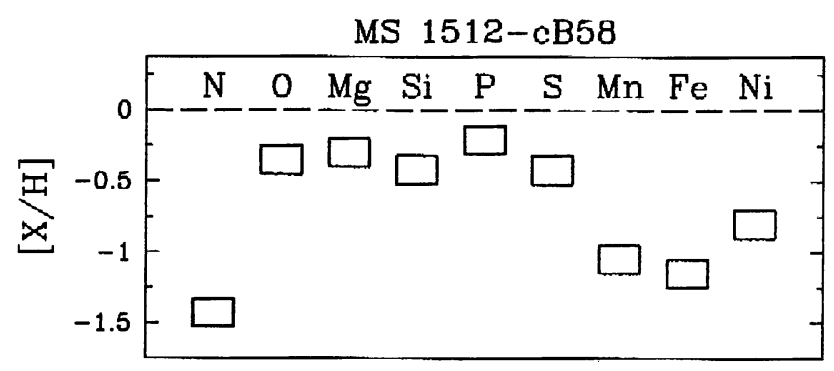

Figure 5. Pattern of chemical abundances in the ambient interstellar medium of MS 1512-cB58. [X/H] denotes the abundance of element X relative to hydrogen compared with the solar value; the scale is logarithmic so that $[\mathrm{X} / \mathrm{H}]=0.0$ is the solar reference and $[\mathrm{X} / \mathrm{H}]=-1$ corresponds to an underabundance by a factor of 10 . The vertical height of the boxes shows the typical uncertainty in the abundance determinations.

\section{Dating the star-formation activity}

Broad-band photometry in the optical and near-infrared, spanning the wavelength interval $900-5500 \AA$ in the rest-frame, is now available for more than one hundred galaxies at $z \simeq 3$ (Papovich, Dickinson \& Ferguson 2001; Shapley et al. 2001). The colours over this range (typically four colours are used in the analysis) depend on the degree of dust reddening, $E(B-V)$, and on the age of the stellar population, $t_{\mathrm{sf}}$. The two can be decoupled to some degree provided that the spectral energy distribution (SED) includes the age-sensitive Balmer break near $3650 \AA$ which at $z=3$ falls between the $H$ - and $K$-bands - hence the need for accurate near-IR photometry. A third parameter, the instantaneous star formation rate, $\Psi\left(t_{\mathrm{sf}}\right)$, determines the normalisation (rather than the shape) of the SED. The analyses by Papovich et al. (2001) and Shapley et al. (2001) deduced the best-fitting values of $E(B-V), t_{\mathrm{sf}}$, and $\Psi\left(t_{\mathrm{sf}}\right)$ by $\chi^{2}$ minimisation of the differences between the observed SEDs and those predicted by the widely used population synthesis code of Bruzual \& Charlot (1993). The results have turned out to be very interesting - some would say surprising (see Figure 6).

Evidently, Lyman break galaxies span a wide range of ages. One fifth of the sample considered by Shapley et al. (2001) consists of objects which seem to have just collapsed and are forming stars on a dynamical timescale ( $\sim 35 \mathrm{Myr})$. At the other end of the scale, some $20 \%$ of the galaxies at $z=3$ have been forming stars for more than $1 \mathrm{Gyr}$, placing the onset of star formation at much higher redshifts $(z>5)$. Furthermore, there appears to be a correlation between age and star formation rate, with the younger objects typically forming stars at about ten times the rate of the older ones and being on average significantly more reddened. The mean SFR is $\left\langle\Psi\left(t_{\mathrm{sf}}\right)\right\rangle=210 h^{-2} \mathrm{M}_{\odot} \mathrm{yr}^{-1}$ for galaxies with $t_{\mathrm{sf}}<35 \mathrm{Myr}$ while, for the $20 \%$ of the sample with $t_{\mathrm{sf}}>1 \mathrm{Gyr},\left\langle\Psi\left(t_{\mathrm{sf}}\right)\right\rangle=25 h^{-2} \mathrm{M}_{\odot} \mathrm{yr}^{-1}$. This range of properties is further reflected in the assembled stellar masses $m_{\text {star }}$ obtained by integrating $\Psi\left(t_{\mathrm{sf}}\right)$ over $t_{\mathrm{sf}}$ (a variety of star formation histories was considered but $m_{\mathrm{star}}$ does not depend sensitively on this choice). As can be seen from Figure 6 , by redshift $z \simeq 3$ some galaxies had apparently already assembled a stellar 

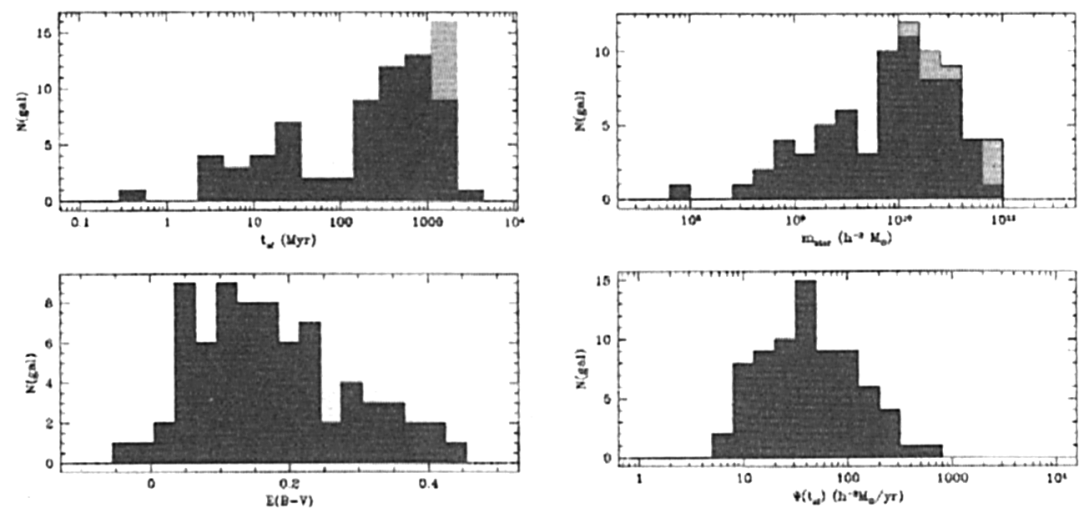

Figure 6. Histograms of best-fitting ages (top left), reddening (bottom left), assembled stellar mass (top right) and star-formation rates (bottom right) for the sample of $81 z \simeq 3$ LBGs analysed by Shapley et al. (2001). There is a large spread of ages in the population; the median age is $320 \mathrm{Myr}$ and $20 \%$ of the objects are older than $1 \mathrm{Gyr}$. The median $E(B-V)=0.155$ for the sample corresponds to attenuations by factors of $\sim 4.5$ and $\sim 2$ at $1600 \AA$ and $5500 \AA$, respectively. By redshift $z \simeq 3$ a significant fraction of LBGs seem to be approaching the stellar mass of today's $L^{*}$ galaxies, $m_{\mathrm{star}} \simeq 4 \times 10^{10} \mathrm{M}_{\odot}$.

mass comparable to that of an $L^{*}$ galaxy today, $m_{\text {star }} \simeq 4 \times 10^{10} \mathrm{M}_{\odot}$, while $20 \%$ of the sample have values of $m_{\mathrm{star}}$ one order of magnitude smaller. These findings led Shapley et al. (2001) to speculate that we may be beginning to discern an evolutionary sequence in Lyman break galaxies, with the younger, dustier, more actively star-forming objects evolving to the older, less reddened, and more quiescent phase.

\section{The impact of massive stars on the intergalactic medium}

During its odyssey from the main sequence to supernova, a massive star deposits large amounts of kinetic energy into the surrounding interstellar medium. The far-UV luminosity of a typical $\left(L^{*}\right) \mathrm{LBG}$ is equivalent to that of 25000007 stars all packed within a projected area with half-light radius of $2-3 \mathrm{kpc}$. When these stars complete their evolution and explode as supernovae, the resultant release of energy drives galactic-scale outflows with speeds of several hundred $\mathrm{km} \mathrm{s}^{-1}$ which can travel to large distances from the sites of star formation. This is a common situation in local starburst galaxies when the star formation rate per unit area exceeds $0.1 \mathrm{M}_{\odot} \mathrm{yr}^{-1} \mathrm{kpc}^{-2}$ (Heckman et al. 2000), and in Lyman break galaxies, where such 'superwinds' have been observed up to $z=5.19$ (Dawson et al. 2002) as systematic velocity offsets between the redshifts of different spectral components. Specifically, the interstellar absorption lines seen against the nuclear starburst are generally blue-shifted relative to the stars and $\mathrm{H}$ II regions, while the resonantly scattered Ly $\alpha$ emission line is normally redshifted by similar amounts. The simplest interpretation is that these two components arise 


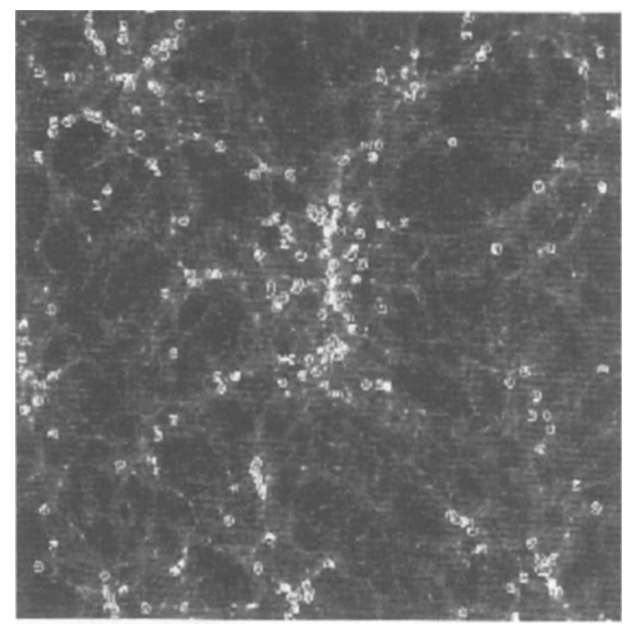

Figure 7. A theorist's view of the high redshift universe. The figure shows a $50 \times 50 \times 5 h^{-3} \mathrm{Mpc}$ slice of the output of an $N$-body simulation of the universe at $z=3$ by Governato et al. (1998). In the large scale structure we see the distribution of dark matter halos which has developed through the growth of primordial density fluctuations. The circles mark the locations of LBGs which are preferentially found in the highest density regions.

in the approaching and receding portions, respectively, of an expanding shell of interstellar matter swept up by the galactic wind.

These outflows have many important consequences, both for the galaxies which host them and for their environments. Since the mass outflow rates are comparable to, or exceed, the star formation rates (e.g., Pettini et al. 2002), they can plausibly provide the regulatory feedback to the process of galaxy formation long invoked by theorists. They can create channels through which Lyman continuum photons from the O- and B-type stars can escape the galaxies (Clarke \& Oey 2002) and ionise the intergalactic medium (Steidel, Pettini \& Adelberger 2001), and have the potential to distribute the products of stellar nucleosynthesis over large volumes (e.g., Aguirre et al. 2001).

Most recently, we have been investigating the impact of these superwinds on the surrounding intergalactic medium (see Figure 7) by comparing the distribution of LBGs with the properties of the Ly $\alpha$ forest seen in absorption against background QSOs in the same fields (Adelberger et al. 2003). One of several surprises produced by this type of analysis is the realisation that the optical depth in the Ly $\alpha$ forest is greatly diminished within a (proper) volume of radius $\sim 100 \mathrm{kpc}$, but otherwise enhanced within $\sim 1 \mathrm{Mpc}$, of the locations of LBGs. Both this 'galaxy proximity effect' and the more general enhancement in the density of $\mathrm{H}$ I where LBGs are found can be explained as the result of the action of superwinds which clear out cavities around the star-forming galaxies and pile up the material in dense shells. It seems inevitable now that our simple ideas of an IGM whose physical conditions are determined solely by the cosmic expan- 
sion and the ionising background will need to be revised to include the effects of massive stars in young galaxies.

\section{Conclusions}

In summary, a picture of the massive star population of Lyman break galaxies is beginning to emerge from a combination of optical and near-IR observations. At redshifts $z \simeq 3$, many of these galaxies already resemble closely what today we call Population I stars in the Milky Way, with a Salpeter slope at the upper end of the IMF and near-solar metallicities. Often, the onset of star formation seems to have occurred well before the time we observe the galaxies; in some cases perhaps at redshifts as high as $z \simeq 10$. Galactic superwinds, driven by the kinetic energy deposited by the star formation activity, are a common feature and probably play a key role in the evolution of LBGs. These outflows of metal-enriched, hot material are also likely to have a significant impact on the intergalactic medium, with far-reaching consequences which we are only now beginning to appreciate fully. In particular, they have the potential for explaining the widespread distribution of metals in the IGM, its reionisation, and its structure over scales of hundreds of kpc. As we learn more about LBGs, the similarity with today's starburst galaxies is as striking as it appeared when they were first discovered (Steidel et al. 1996). Thus the link between observational cosmology and stellar astrophysics remains a strong one. In particular, it is clear from this brief review that future advances in the modelling of young stellar populations and in the tools at our disposal for interpreting their spectra will feed directly into a much improved understanding of some of the earliest galaxies in the universe.

\section{References}

Adelberger, K.L., Steidel, C.C., Shapley, A.E., Pettini, M. 2003, ApJ 584, 45

Aguirre, A., Hernquist, L., Schaye, J., Weinberg, D. H., Katz, N., Gardner, J. 2001, ApJ 560, 599

Bruzual, G.A., Charlot, S. 1993, ApJ 405, 538

Clarke, C.J., Oey, M.S. 2002, MNRAS 337, 1299

Dawson, S., Spinrad, H., Stern, D., Dey, A., van Breugel, W., de Vries, W., Reuland, M. 2002, ApJ 570, 92

Governato, F., Baugh, C.M., Frenk, C.S., Cole, S., Lacey, C.G., Quinn, T., Stadel, J. 1998, Nature 392, 359

Heckman, T.M., Lehnert, M.D., Strickland, D.K., Armus, L. 2000, ApJS 129, 493

Holweger, H., 2001, in: R.F. Wimmer-Schweingruber (ed.), Solar and Galactic Composition, AIP-CP 598, 23

Hu, E.M., Cowie, L.L., McMahon, R.G., Capak, P., Iwamuro, F., Kneib, J.-P., Maihara, T., Motohara, K. 2002, ApJ (Letters) 568, L75

Leitherer, C., Schaerer, D., Goldader, J.D., et al. 1999, ApJS 123, 3

Leitherer, C., Leão, J.R.S., Heckman, T.M., Lennon, D.J., Pettini, M., Robert, C. 2001, ApJ 550, 724

Papovich, C., Dickinson, M., Ferguson, H.C. 2001, ApJ 559, 620 
Pettini, M., Rix, S.A., Steidel, C.C., Adelberger, K.L., Hunt, M.P., Shapley, A.E. 2002, ApJ 569, 742

Pettini, M., Shapley, A.E., Steidel, C.C., Cuby, J.-G., Dickinson, M., Moorwood, A.F.M., Adelberg, K.L., Giavalisco, M. 2001, ApJ 554, 981

Pettini, M., Steidel, C.C., Adelberger, K.L., Dickinson, M., Giavalisco, M. 2000, ApJ 528, 96

Shapley, A.E., Steidel, C.C., Adelberger, K.L., Dickinson, M., Giavalisco, M., Pettini, M. 2001, ApJ 562, 95

Shapley, A.E., Steidel, C.C., Adelberger, K.L., Pettini, M., 2003 ApJ in press

Steidel, C.C. 2000, in: J. Bergeron (ed.), Discoveries and Research Prospects from 8- to 10-Meter-Class Telescopes, Proc. SPIE 4005, 22

Steidel, C.C., Giavalisco, M., Pettini, M., Dickinson, M., Adelberger, K.L. 1996, ApJ (Letters) 462, L17

Steidel, C.C., Pettini, M., Adelberger, K.L. 2001, ApJ 546, 665

Steidel, C.C., Pettini, M., Hamilton, D. 1995, AJ 110, 2519

\section{Discussion}

KUDRITZKI: You obtain 1/10 galactic Fe-abundance in cB 58 and 1/3 for $\alpha$-elements form the ISM absorption lines. While the $1 / 3 \mathrm{CNO}$ is confirmed by the integrated stellar C IV line, I wonder whether the iron features in the integrated stellar spectra confirm $1 / 10$ solar. And, what is the amount of depletion of metals in dust?

PETtini: There is likely to be some depletion of the Fe-peak elements onto dust (which we know to be present in MS1512-cB58), but this probably accounts for only part, maybe half, of the observed underabundances. The factor of $\sim 2$ intrinsic underabundance of $\mathrm{Fe}$ (due to the delay in its release from Type Ia SNe) will not have a large effect on the equivalent width of the $\lambda 1425$ blend as $\mathrm{Fe} v \lambda 1430$ contributes only $\sim 1 / 3$ to the blend.

KUDRITZKI: The galactic outflow in MS 1512-cB58 is something like a giant stellar wind bubble. I wonder, whether the simple estimate has been made to check if the wind energy of $10^{5} \mathrm{O}$-type stars is enough to drive the bubble.

Pettini: Tim Heckman has looked at this in some detail and concluded that less $\lesssim 10 \%$ of the kinetic energy delivered by the starburst is required to drive these large scale outflows.

HILLIER: You are now measuring abundances in the Lyman-break galaxies at the factorof-2 level. At this level you need to be concerned with the definition of the abundance scale. CNO abundances in the Sun seem to be 0.2-0.3 dex smaller than in O- and Btype stars in the solar neighborhood. While the absolute scale may not be important when using differential measurements, it is important for constraining synthesis models, and may be important for the predictions of ionizing fluxes.

PETtinI: I agree, John. Maybe one of these days we will have a more reliable solar abundance scale, especially for C, N and O! The recent revision by Holweger (2001) goes some way towards resolving the discrepancy, at least for oxygen. 